\title{
Evaluating a CALL software on the learning of English prepositions
}

\author{
Bing Hiong Ngu ${ }^{\mathrm{a}, *}$, Soubakeavathi Rethinasamy ${ }^{\mathrm{b}}$ \\ ${ }^{a}$ Faculty of Cognitive Sciences and Human Development, Universiti Malaysia Sarawak, Kota Samarahan, \\ 94300 Kota Samarahan, Sarawak, Malaysia \\ ${ }^{\mathrm{b}}$ English Language Centre, Universiti Malaysia Sarawak, 94300 Kota Samarahan, Sarawak, Malaysia
}

Received 15 August 2002; accepted 18 August 2004

\begin{abstract}
This study assessed the effectiveness of using a CALL lesson (Computer Assisted Language Learning) over a conventional lesson to facilitate learning of English prepositions at Bario, Malaysia. CALL was developed by the Ministry of Education, Malaysia as support material to enhance learning of English prepositions. Both the conventional and the CALL lessons were matched with the same content except for the medium in which the lesson was being delivered. Students were provided with computers to go through the CALL lesson in a self-regulated manner; while a teacher taught the conventional lesson in a classroom. Test results indicate that students who received the conventional lesson outperformed those who went through the CALL lesson. The Relative Condition Efficiency measurement also showed that the conventional group learned more efficiently than the CALL group. The findings are interpreted from the perspective of cognitive load required in processing the presentation mode of the learning materials.
\end{abstract}

(c) 2004 Published by Elsevier Ltd.

Keywords: Evaluation methodologies; Evaluation of CALL systems; Human-computer interface; Cognitive load; Secondary education

\footnotetext{
${ }^{*}$ Corresponding author.

E-mail address: bhngu@fcs.unimas.my (B.H. Ngu).
} 